\title{
REMARKS ON THE STABILITY OF SHOCK PROFILES FOR CONSERVATION LAWS WITH DISSIPATION
}

BY

\author{
ROBERT L. PEGO
}

\begin{abstract}
Two remarks are made. The first is to establish the stability of monotone shock profiles of the KdV-Burgers equation, based on an energy method of Goodman. The second remark illustrates, specifically in Burgers' equation, that uniform rates of decay are not to be expected for perturbations of shock profiles in typical norms.
\end{abstract}

Introduction. We will prove two results. The first is to establish the orbital stability of monotone shock profile solutions $\phi(x-s t)$ of the $\mathrm{KdV}$-Burgers equation

$$
u_{t}+u u_{x}-\mu u_{x x}-\nu u_{x x x}=0, \quad \mu>0 .
$$

The second is to show that for shock profile solutions of viscous conservation laws, and specifically for Burgers' equation

$$
u_{t}+u u_{x}-\mu u_{x x}=0,
$$

one cannot expect perturbations to decay at any uniform rate (algebraic or otherwise) in ordinary norms, such as $L^{p}$.

The first result is related to Goodman's conditional stability result in [5] for small amplitude shock profiles $\phi(x-s t)$ in systems of genuinely nonlinear conservation laws with viscosity, $u_{t}+f(u)_{x}=B u_{x x}, u \in \mathbf{R}^{m}$. The second is intended to be relevant to the question of full stability for shock profiles of such systems. The nonlinear stability to small perturbations of constant state solutions $u_{0}(x) \equiv c$ of the system $u_{t}+f(u)_{x}=\left(B(u) u_{x}\right)_{x}$, where $B(c)$ is a strictly stable viscosity matrix (see [9]), can be demonstrated based on a uniform rate of decay of solutions to the linearized equation. The method used is similar to that developed in [8] and [10] for systems in $n>1$ space dimensions. Faster decay rates in higher space dimensions make the method easier to apply when $n$ is large, but it does just work for conservation laws with stable viscosities in one space dimension (see [13]). Thus, for example, assuming $c=0$, if $\left\|u_{0}\right\|_{H^{6} \cap L^{1}}$ is sufficiently small, then $\|u(\cdot, t)\|_{H^{4}} \leqslant$ $C t^{-1 / 4}\left\|u_{0}\right\|_{H^{4} \cap L^{1}}$.

In this note, we want to point out that such uniform decay rates cannot be valid for perturbations of shock profiles. In particular, for the shock profile solution of Burgers' equations we construct a family of perturbations which decay to zero but do so at no uniform rate in most ordinary norms (including all $L^{p}$ norms). Thus, for

Received by the editors October 15, 1984 and, in revised form, December 19, 1984.

1980 Mathematics Subject Classification. Primary 35Q20, 35L65. 
example, perturbations of shock profiles do not decay at the same uniform rate as perturbations of constant states.

2. Stability of KdV-Burgers' shock profiles. We consider monotone shock profile solutions $\phi(x-s t)$ of the KdV-Burgers equation (1.1). Such a profile satisfies

$$
\begin{gathered}
\nu \phi^{\prime \prime \prime}+\mu \phi^{\prime \prime}-(\phi-s) \phi^{\prime}=0, \\
\phi \rightarrow u_{l} \text { as } x \rightarrow-\infty, \\
\phi \rightarrow u_{r} \text { as } x \rightarrow+\infty
\end{gathered}
$$

The profile exists provided that $u_{l}, u_{r}$, and $s$ satisfy $s=\left(u_{l}+u_{r}\right) / 2$ and $u_{l}>u_{r}$. The profile is monotone if and only if $\mu \geqslant \sqrt{2|\nu|\left(u_{l}-u_{r}\right)}$. (See [1] for a discussion of existence of these profiles.) Applying the transformations

$$
x \leftarrow x-s t, \quad t \leftarrow c t, \quad u \leftarrow(u-s) / c, \quad \mu \leftarrow \mu / c, \quad \nu \leftarrow \nu / c,
$$

where $c=\left(u_{l}-u_{r}\right) / 2$, we may assume $u_{l}=1, u_{r}=-1, s=0$, and $\mu \geqslant 2 \sqrt{|\nu|}$. Then the profile $\phi(x)$ is a stationary solution of $(1.1)$.

We shall discuss solutions of the form $u(x, t)=\phi(x)+v(x, t)$, where $v(\cdot, t)$ is in some space of integrable functions. The perturbation $v$ must satisfy

$$
\begin{aligned}
& v_{t}=\nu v_{x x x}+\mu v_{x x}-(\phi v)_{x}-\frac{1}{2}\left(v^{2}\right)_{x}, \\
& v(x, 0)=v^{0}(x) .
\end{aligned}
$$

Asymptotic stability of the profile $\phi(x)$ means that any perturbation $v$ decays to zero as $t \rightarrow \infty$. Of course, any translate $\phi(x-h)$ of the profile is also a stationary solution of (1.1), so $v(x)=\phi(x-h)-\phi(x)$ is a stationary solution of (2.3) and does not decay to zero. In general, however, we can expect that $u(x, t)-\phi(x-h)$ will decay to zero for some shift $h$ (whence the term orbital stability above). This shift is determined by the requirement that $\int_{-\infty}^{\infty}(u(x, 0)-\phi(x-h)) d x=0$ if $v^{0}(x)$ is integrable. (Note that one expects $\int v(x, t) d x$ to be constant in time.) Thus, we just assume $h=0$ and require $\int v^{0}(x) d x=0$ for integrable $v^{0}$. In the $L^{2}$ theory below, we require $v^{0}=V_{x}^{0}$ instead, where $V^{0} \in H^{1}$. Here $H^{j}$ denotes the Sobolev space of functions differentiable $j$ times in $L^{2}(\mathbf{R})$, where the norm

$$
\|u\|_{H^{j}}=\left(\sum_{k=0}^{j} \int\left|D^{k} u\right|^{2}\right)^{1 / 2} .
$$

$C\left([0, T), H^{j}\right)$ denotes the space of continuous functions on $[0, T)$ with values in $H^{j}$.

TheOrem 2.1. Assume $v^{0}(x)=V_{x}^{0}(x)$, where $V^{0} \in H^{3}$. If $\left\|V^{0}\right\|_{H^{3}}$ is sufficiently small, then a solution $v(x, t)$ to $(2.3)$ exists in $C\left([0, \infty), H^{2}\right)$ and satisfies $\|v(t)\|_{H^{2}} \rightarrow 0$ as $t \rightarrow \infty$.

The proof is by an $L^{2}$ energy method, using an idea of Goodman [5]. We seek a solution of $(2.3)$ in the form $v(x, t)=V_{x}(x, t)$, where $V(x, t)$ in $C\left([0, \infty), H^{3}\right)$ satisfies the equation

$$
V_{t}=\nu V_{x x x}+\mu V_{x x}-\phi V_{x}-\frac{1}{2} V_{x}^{2}, \quad V(x, 0)=V^{0}(x) .
$$


Global existence and decay for $v(x, t)$ will be derived from a local existence theorem for $V$ combined with the time-uniform estimate

$$
\|V(t)\|_{H^{3}}^{2}+\int_{0}^{t}\|D V(s)\|_{H^{2}}^{2} d s \leqslant C_{1}\left\|V^{0}\right\|_{H^{3}}^{2} \text { for } 0 \leqslant t<T
$$

where $C_{1}$ is independent of $T$, if $\|V(t)\|_{H^{3}}$ is sufficiently small for $0 \leqslant t \leqslant T$.

As the first step, we establish estimate (2.5), assuming that the solution $V$ to (2.4) is smooth, say in $C^{1}\left([0, T), H^{s}\right)$ with $s$ large. Compute

$$
\begin{aligned}
\frac{d}{d t} \int V^{2}(x, t) d x & =2 \int V\left(\nu V_{x x x}+\mu V_{x x}-\phi V_{x}-\frac{1}{2} V_{x}^{2}\right) \\
& =-\int(2 \mu+V) V_{x}^{2}+\int \phi_{x} V^{2} .
\end{aligned}
$$

In the last term, we use the equality $2 \int V \phi V_{x}=-\int \phi_{x} V^{2}$ to symmetrize the derivatives on $V$. Hence

$$
\frac{d}{d t} \int V^{2}(x, t) d x+2 \mu \int V_{x}^{2}-\int \phi_{x} V^{2} \leqslant\|V\|_{\infty} \int V_{x}^{2}
$$

The monotonicity of the profile $\phi$ implies $\phi_{x}<0$. Thus, if $\|V\|_{\infty}$ is sufficiently small, note that $\left(\mathrm{E}_{0}\right)$ implies that $\|V(t)\|_{L^{2}}^{2}$ is decreasing in time.

We now estimate higher derivatives, computing, for $j=1,2,3$,

$$
\frac{d}{d t} \int\left|D^{j} V(x, t)\right|^{2} d x=2 \int D^{j} V D^{j}\left(\nu V_{x x x}+\mu V_{x x}-\phi V_{x}-\frac{1}{2} V_{x}^{2}\right) .
$$

By integrating by parts and symmetrizing derivatives on $V$, one easily verifies explicitly that, if $j=1,2,3$,

$$
\begin{gathered}
-2 \int D^{j} V D^{j}\left(\phi V_{x}\right) \leqslant C_{\phi} \sum_{k=1}^{j} \int\left|D^{k} V\right|^{2} \\
-\int D^{j} V D^{j}\left(V_{x}^{2}\right)=C_{j} \int V_{x x}\left|D^{j} V\right|^{2} \leqslant c\left\|V_{x x}\right\|_{\infty} \int\left|D^{j} V\right|^{2} .
\end{gathered}
$$

Hence, for $j=1,2,3$,

$$
\begin{aligned}
& \frac{d}{d t} \int\left|D^{j} V\right|^{2}(t)+2 \mu \int\left|D^{j+1} V\right|^{2} \\
& \leqslant c\left\|V_{x x}\right\|_{\infty} \int\left|D^{j} V\right|^{2}+C_{\phi} \sum_{k=1}^{j} \int\left|D^{k} V\right|^{2} .
\end{aligned}
$$

Take $\tilde{\mu} \leqslant 1$ (to be fixed in a moment). Multiply $\left(\mathrm{E}_{j}\right)$ by $\tilde{\mu}^{j}, j=0,1,2,3$, and add to obtain

$$
\begin{aligned}
& \frac{d}{d t} \sum_{j=0}^{3} \tilde{\mu}^{j} \int\left|D^{j} V\right|^{2}+2 \mu \sum_{j=0}^{3} \tilde{\mu}^{j} \int\left|D^{j+1} V\right|^{2} \\
& \quad \leqslant\|V\|_{\infty} \int V_{x}^{2}+\tilde{\mu}\left(c\left\|V_{x x}\right\|_{\infty}+3 C_{\phi}\right) \sum_{j=1}^{3} \tilde{\mu}^{j-1} \int\left|D^{j} V\right|^{2} .
\end{aligned}
$$


Now fix $\tilde{\mu}<\mu / 3 C_{\phi}$. As long as

$$
\|V\|_{\infty}+c \tilde{\mu}\left\|V_{x x}\right\|_{\infty} \leqslant \mu-3 \tilde{\mu} C_{\phi},
$$

inequality (E) implies

$$
\frac{d}{d t} \sum_{j=0}^{3} \tilde{\mu}^{j} \int\left|D^{j} V\right|^{2}+\mu \sum_{j=0}^{3} \tilde{\mu}^{j} \int\left|D^{j+1} V\right|^{2} \leqslant 0 .
$$

Since the left side of (2.6) is dominated by $\|V\|_{H^{3}}$, one may choose $\varepsilon_{1}>0$ so that (2.6) holds if $\|V\|_{H^{3}}<\varepsilon_{1}$. Then (2.7) holds, and integration in $t$ establishes the time-uniform estimate (2.5).

REMARK 1. At this point we shall also observe that if $\|V\|_{H^{3}}<\varepsilon_{1}$, then the quantities $\int V^{2}(x, t) d x$ and $\int V^{2}+\sum_{j=1}^{3} \tilde{\mu}^{j} \int\left|D^{j} V\right|^{2}$ are nonincreasing functions of $t$. For smooth solutions, this follows from $\left(\mathrm{E}_{0}\right)$ and $(\mathrm{E})$ with (2.6). (We will need this remark to establish the decay of the perturbation $v$ in (2.3).)

The proof of Theorem 2.1 will combine (2.5) with the local existence theorem (Theorem 2.2). It is inappropriate to give a full proof of Theorem 2.2 here. Instead, we remark that the proof can be constructed exactly along the line followed in [3] for $H^{s}$ solutions of the KdV-Burgers equation. Also see [2].

Theorem 2.2. Assume $V^{0}(x) \in H^{s}, s \geqslant 3$. There exists $T_{0}>0$ such that a unique solution $V(x, t)$ to $(2.4)$ exists in $C\left(\left[0, T_{0}\right], H^{s}\right)$ and depends continuously on $V^{0}$ in $H^{s}$. $T_{0}$ may be chosen to depend solely on $\left\|V^{0}\right\|_{H^{3}}$, decreasing as $\left\|V^{0}\right\|_{H^{3}}$ increases. Then there also exists $C_{0}$ such that

$$
\|V(t)\|_{H^{3}} \leqslant C_{0}\left\|V^{0}\right\|_{H^{3}}
$$

for $0 \leqslant t \leqslant T_{0}$ ( provided $\left\|V^{0}\right\|_{H^{3}} \leqslant M$ for some $\left.M\right)$.

We now finish the proof of Theorem 2.1. First, we remark that Theorem 2.2 can be strengthened to conclude that for every $t>0, V(t)$ is in $H^{s}$ for all $s$. It now follows easily that estimate (2.5) and Remark 1 are valid for solutions of (2.4) which are merely in $C\left([0, T], H^{3}\right)$, since $V(t+\varepsilon) \rightarrow V(t)$ and, for $t \geqslant 0, V(t+\varepsilon)$ is smooth.

The next step is to establish the global existence and boundedness of small solutions in $C\left([0, \infty), H^{3}\right)$ based on Theorem 2.2 and estimate (2.5), which is now valid for such solutions. Fix $\varepsilon_{1}>0$ and $C_{1} \geqslant 1$ so that (2.5) holds if $\|V\|_{H^{3}}<\varepsilon_{1}$. By Theorem 2.2, choose $T_{0}>0$ and $C_{0} \geqslant 1$ so that 2.2 applies, with this $T_{0}, C_{0}$, for $\left\|V^{0}\right\|_{H^{3}} \leqslant \varepsilon_{1}$.

Fix $\varepsilon_{0}>0$ so that $C_{0} C_{1} \varepsilon_{0}<\varepsilon_{1}$. For all $T \geqslant 0$, we claim that if $\left\|V^{0}\right\|_{H^{3}} \leqslant \varepsilon_{0}$, then the solution $V \in C\left([0, T], H^{3}\right)$ and $\|V(t)\|_{H^{3}} \leqslant C_{1} \varepsilon_{0}<\varepsilon_{1}$ for all $t$.

This claim holds trivially for $T=0$. Assume it holds for a fixed $T \geqslant 0$; we claim it follows for $T+T_{0}$. Indeed, we assume that $\left\|V^{0}\right\|_{H^{3}} \leqslant \varepsilon_{0}$ and that $V \in C\left([0, T], H^{3}\right)$, with $\|V(t)\|_{H^{3}} \leqslant C_{1} \varepsilon_{0}$ for $0 \leqslant t \leqslant T$.

Applying the local existence theorem at time $T$, we extend the solution $V$ to $C\left(\left[0, T+T_{0}\right], H^{3}\right)$, and (2.8) implies

$$
\|V(t)\|_{H^{3}} \leqslant C_{0}\|V(T)\|_{H^{3}} \leqslant C_{0} C_{1} \varepsilon_{0}<\varepsilon_{1} \quad \text { for } T \leqslant t \leqslant T+T_{0} .
$$


But then estimate (2.5) holds on the entire interval $\left[0, T+T_{0}\right]$; hence,

$$
\|V(t)\|_{H^{3}} \leqslant C_{1} \varepsilon_{0} \text { for } 0 \leqslant t \leqslant T+T_{0} .
$$

The claim now follows for all $T \geqslant 0$.

The global existence and boundedness of the solution have been established, provided $\left\|V^{0}\right\|_{H^{3}} \leqslant \varepsilon_{0}$. We now show that the original perturbation $v=V_{x}$ decays to zero in $H^{2}$ as $t \rightarrow \infty$. By Remark 1 we know that $\int V^{2}(x, t) d x$ and $\int V^{2}(x, t) d x+$ $\sum_{j=1}^{3} \tilde{\mu}^{j} \int\left|D^{j} V\right|^{2}$ are nonincreasing in time; hence, they approach limits as $t \rightarrow \infty$. Then $\sum_{j=1}^{3} \tilde{\mu}^{j} f\left|D^{j} V\right|^{2}$ approaches a limit as $t \rightarrow \infty$, and since this quantity is integrable over $[0, \infty)$ by $(2.5)$, it follows that the limit is zero, so

$$
\|v(t)\|_{H^{2}}^{2}=\sum_{j=1}^{3} \int\left|D^{j} V\right|^{2} \rightarrow 0 \quad \text { as } t \rightarrow \infty .
$$

This completes the proof of Theorem 2.1.

We remark that this proof of stability of the shock profile does not yield a decay rate for the perturbation $v$, except in the sense that $\int_{0}^{\infty}\|v(t)\|_{H^{2}}^{2} d t \leqslant C_{1}\left\|V^{0}\right\|_{H^{3}}^{2}$.

3. Nonuniform decay in Burgers' equation. Several works have demonstrated the orbital stablity of shock profiles in Burgers' equation (1.2) (see [7, 11, 12]). Indeed, Theorem 2.1 is valid in the case $\nu=0$. Many approaches to questions of asymptotic stability, including those in $[\mathbf{8}, 10,11,12]$, use a decay rate to establish nonlinear stability. Our goal in this section is to show that for perturbations of shock profiles in Burgers' equation which decay to zero, uniform decay rates cannot be obtained in "ordinary" norms, such as $L^{p}$ norms.

Let $\phi(x-s t)$ be a shock profile solution of Burgers' equation (1.2). After scaling, we may assume $s=0, \mu=1$. Writing $u(x, t)=\phi(x)+v(x, t)$, the perturbation $v$ satisfies

$$
v_{t}=v_{x x}-(\phi v)_{x}-\frac{1}{2}\left(v^{2}\right)_{x}, \quad v(x, 0)=v^{0}(x) .
$$

We shall construct a family of perturbations which do decay to zero, but at no uniform rate, measured in "typical" norms. The idea is to start with a stationary perturbation $\Phi_{\gamma}(x)=\phi(x-\gamma)-\phi(x), \gamma \neq 0$, which has the effect of shifting the profile's phase. Of course, $\int_{-\infty}^{\infty} \Phi_{\gamma}(x) d x \neq 0$, and this perturbation does not decay to zero. Therefore, we add a compensating mass to the initial data and consider a perturbation $v$ satisfying (3.1), with

$$
v^{0}(x)=\Phi_{\gamma}(x)-\Phi_{\gamma}(x-L) .
$$

Now $\int_{-\infty}^{\infty} v^{0}(x) d x=0$, and this perturbation will decay to zero. But the shift $L$ may be chosen arbitrarily large, so it may be an arbitrarily long time before the nearly stationary part of the solution in the region dominated by $\Phi_{\gamma}(x)$ is seriously affected. With respect to any norm in which the contributions of the $\Phi_{\gamma}(x)$ and $\Phi_{\gamma}(x-L)$ terms are roughly equal, one expects that it could take an arbitrarily long time for the norm of the solution to decay by more than about half.

Although this sort of reasoning might be more widely applied, we shall give a rigorous result only for Burgers' equation. We consider functional norms || \| 
satisfying three conditions (e.g., $L^{p}$ norms):

(1) $\|u(\cdot-\gamma)\|=\|u(\cdot)\|$ for all real $\gamma$ and functions

$$
u(x) \text { (translation invariance); }
$$

(2) $|v(x)| \leqslant|u(x)|$ a.e. implies $\|v\| \leqslant\|u\|$ (Banach norm);

(3) $\|u\|$ is finite if $u(x)$ is smooth and rapidly decreasing.

Let \|\|$_{1}$ and \|\|$_{2}$ be any two such norms. (The solution and initial data may be measured in different norms.) A uniform decay rate for (3.1) is a positive decreasing function $K(t)$ defined for $t>0$, with $K(t) \rightarrow 0$ as $t \rightarrow \infty$, such that, for any smooth solution of (3.1) with $\int_{-\infty}^{\infty} v^{0}(x) d x=0$, we have

$$
\|v(\cdot, t)\|_{2} \leqslant K(t)\left\|v^{0}\right\|_{1} .
$$

Proposition 3.1. If the norms \|\|$_{1}$ and \|\|$_{2}$ satisfy (3.2), then there is no uniform decay rate for (3.1).

REMARK 2. This construction also rules out uniform decay rates in $H^{s}$, as established for perturbations of constant states in [13]. However, it does not preclude a decay estimate of the form $\|v(t)\|_{L^{2}} \leqslant C t^{-\alpha}\left\|V^{0}\right\|_{L^{2}}$, where $V_{x}^{0}=v(0)$. (Goodman probably means to conjecture this sort of estimate in [5].)

We may assume $\phi(x) \rightarrow 1$ as $x \rightarrow \infty, \phi(x) \rightarrow-1$ as $x \rightarrow-\infty$. The shock profile is given explicitly by $\phi(x)=-\tanh (x / 2)=2 /\left(1+e^{x}\right)-1$. Fix any $\gamma>0$. Then $\Phi_{\gamma}(x)=\phi(x-\gamma)-\phi(x)$ is a stationary solution of (3.1) and is smooth, positive and exponentially decreasing as $|x| \rightarrow \infty$, with maximum value

$$
\left(1+\frac{2 \sinh \gamma / 2}{1+\cosh \gamma / 2}\right) \text { at } x=\frac{\gamma}{2} \text {. }
$$

Let $\varepsilon>0$. Fix $\Psi(x)$ in $C_{0}^{\infty}(\mathbf{R})$ so that

$$
\Psi(x) \geqslant 0 \text { and } \Phi_{\gamma}(x)-2 \varepsilon<\Psi(x)<\max \left\{\Phi_{\gamma}(x)-\varepsilon, 0\right\} \text { for all } x .
$$

In (3.1) choose

$$
v^{0}(x)=\Phi_{\gamma}(x)-\Phi_{\gamma}(x-L) .
$$

If $L$ is large enough, $\left|v^{0}(x)\right| \geqslant \Psi(x)+\Psi(x-L) \geqslant \Psi(x)$ for all $x$, so $\left\|v^{0}\right\|_{1} \geqslant\|\Psi\|_{1}$ for $L$ large. Also, $\left\|v^{0}\right\|_{1} \leqslant 2\left\|\Phi_{\gamma}\right\|_{1}$ by (3.2). The function $\Psi(x)$ will be used as a convenient lower bound for the perturbation.

Proposition 3.2. Fix any $T>0$. If $L$ is sufficiently large, then the solution of (3.1) with initial data (3.3) satisfies $|v(x, T)| \geqslant \Psi(x)$ for all $x$.

Given this proposition, we easily complete the proof of Proposition 3.1. Assume a uniform decay rate $K(t)$ exists. Thus, $\|v(\cdot, t)\|_{2} \leqslant K(t)\left\|v^{0}\right\|_{1} \leqslant 2 K(t)\left\|\Phi_{\gamma}\right\|_{1}$ for all $t>0$. Choose $T$ so large that $\|\Psi\|_{2}>2 K(T)\left\|\Phi_{\gamma}\right\|_{1}$. But if $L$ is sufficiently large, then Proposition 3.2 and (3.2) imply that $\|v(\cdot, T)\|_{2} \geqslant\|\Psi\|_{2}$, yielding a contradiction. Hence, no uniform decay rate exists.

In turn, Proposition 3.2 is a consequence of a simple "finite speed of substantial propagation" property, which we state in Proposition 3.3 below. Indeed, suppose $|\Psi(x)|=0$ if $|x|>R_{1}$. Then Proposition 3.2 is implied by the estimate

$$
\left|v(x, T)-\Phi_{\gamma}(x)\right|<\varepsilon \text { for }|x|<R_{1},
$$


for the difference of the two solutions $v$ and $\Phi_{\gamma}$ of (3.1). By Proposition 3.3 this estimate is valid if $L$ is sufficiently large, for if $R_{0}>0$ and $\delta>0$ are fixed from 3.3, then

$$
\left|v^{0}(x)-\Phi_{\gamma}(x)\right|<\delta \text { for }|x|<R_{0} \text {, if } L \text { is large. }
$$

Proposition 3.3. Let $v_{1}(x, t)$ and $v_{2}(x, t)$ be two solutions of $(3.1)$, with $v_{i}(x, 0)$ bounded, continuous, and in $L^{1}, i=1,2$. Fix $T, R_{1}$, and $\varepsilon$ positive. There exist $R_{0}$ and $\delta>0$ so that, if

$$
\left|v_{1}(x, 0)-v_{2}(x, 0)\right|<\delta \text { for }|x|<R_{0},
$$

then

$$
\left|v_{1}(x, T)-v_{2}(x, T)\right|<\varepsilon \text { for }|x|<R_{1} .
$$

For completeness we give the straightforward proof of Proposition 3.3 based on the Hopf-Cole transformation, which linearizes Burgers' equation (1.2). We take $\mu=1$. Global existence, regularity, and uniqueness of the solution of Burgers' equation for, say, bounded and continuous initial data follow from the results of Hopf [6]. Let $u^{0}(x)=\phi(x)+v^{0}(x)$ be given, with $v^{0}$ continuous and integrable. Using the classical heat kernel, solve the initial value problem

$$
\begin{aligned}
& w_{t}=w_{x x}, \quad t>0, \\
& w(x, 0)=\exp -\frac{1}{2} \int_{0}^{x} u^{0}(y) d y .
\end{aligned}
$$

Define $u(x, t)=-2 w_{x} / w$ and $v=u-\phi$. From [6] it follows that $u(x, t)$ is continuous for $t \geqslant 0$, smooth for $t>0$, satisfies (1.2), and

$$
\begin{aligned}
w(x, t) & =\exp -\frac{1}{2} \int_{0}^{x} u(y, t) d y \cdot \exp -\frac{1}{2} \int_{0}^{t}\left(u_{x}-\frac{1}{2} u^{2}\right)(0, s) d s \\
& \equiv H u(x, t) .
\end{aligned}
$$

When $v \equiv 0$, we compute explicitly that

$$
H \phi(x, t)=e^{-t / 4} \exp \int_{0}^{x}\left(\frac{1}{2}-\frac{1}{1-e^{y}}\right) d y=e^{-t / 4} \cosh \frac{x}{2} .
$$

Since $\phi(0)=0, H(\phi+v)=H \phi H v$. Letting $z(x, t)=H v$, one checks that $z=$ $e^{t / 4} \operatorname{sech}(x / 2) w$ is a solution of

$$
\begin{aligned}
& z_{t}=z_{x x}-\phi z_{x}, \quad t>0, \\
& z(x, 0)=\exp -\frac{1}{2} \int_{0}^{x} v^{0}(y) d y,
\end{aligned}
$$

and that $v=u-\phi$ is the solution of (3.1) and satisfies $v=-2 z_{x} / z$. Explicitly, the solution of (3.5) is given (by transformation to $w$ ) by

$$
z(x, t)=\int_{-\infty}^{\infty} K(x, y, t) z(y, 0) d y,
$$

where

$$
K(x, y, t)=\frac{1}{\sqrt{4 \pi t}} \exp \frac{-(x-y)^{2}}{4 t} \frac{\cosh y / 2}{\cosh x / 2} .
$$


In order to prove Proposition 3.3, let $v_{1}(x, t)$ and $v_{2}(x, t)$ be two regular solutions of (3.1), with $v_{i}(x, 0)$ bounded, continuous and integrable, and let $z_{i}=H v_{i}$. Then

$$
v_{1}-v_{2}=\frac{1}{z_{1}}\left(\left(z_{1}-z_{2}\right)_{x}+\left(z_{2}-z_{1}\right) \frac{z_{2 x}}{z_{2}}\right) \text {. }
$$

Using this relation, we can reduce Proposition 3.3 to an analogous result for the simpler (3.5). However, we must first establish some bounds.

Lemma 3.4. There exist positive $m$ and $M$, depending on $\max \left\|v_{i}(x, 0)\right\|_{L^{1} \cap L^{\infty}}$, so that

$$
0<m \leqslant z_{1}(x, t) \leqslant M \text { and }\left|v_{2}(x, t)\right| \leqslant M
$$

for all $x, 0 \leqslant t \leqslant T$.

Proof. Clearly, we may choose $m$ and $M$ so that, for $t=0,0<m \leqslant z_{1} \leqslant M$ and $|u(x, t)| \leqslant M-1$. By applying a suitable maximum principle to (1.2) and (3.5), these estimates will remain valid for all $t>0$. For instance, the estimate for $z_{1}$ is easily established by using the explicit kernel $K$ for (3.5), which is positive and must satisfy $\int K(x, y, t) d y=1$ for all $t>0$ and all $x$. The fact that the maximum principle is valid for solutions of Burgers' equation with bounded initial data follows from the results of [4] and [6]. Our lemma follows.

Using Lemma 3.4 and (3.6), one sees that

$$
\left|\left(v_{1}-v_{2}\right)(x, T)\right| \rightarrow 0 \text { as }\left|D^{j}\left(z_{1}-z_{2}\right)(x, T)\right| \rightarrow 0
$$

for $j=0$ and 1 , uniformly for $|x| \leqslant R_{1}$, and that, given $R_{0}>0,\left|D^{j}\left(z_{1}-z_{2}\right)(x, 0)\right|$ $\rightarrow 0$ as $\left|\left(v_{1}-v_{2}\right)(x, 0)\right| \rightarrow 0$ uniformly for $|x| \leqslant R_{0}$. Observe that $z=z_{1}-z_{2}$ is a solution of (3.5) with $|z| \leqslant M$. Proposition 3.3 is implied by Lemma 3.5, which supplies the "finite speed of substantial propagation" property for (3.5).

Lemma 3.5. Let $\varepsilon_{1}>0$. There exist $R_{0}, \delta_{1}>0$, so that if $z=z_{1}-z_{2}$ satisfies $\left|D^{j_{z}}(x, 0)\right| \leqslant \delta_{1}$ for $j=0$ and 1 , and $|x| \leqslant R_{0}$, then $\left|D^{j_{z}}(x, T)\right| \leqslant \varepsilon_{1}$ for $|x| \leqslant R_{1}$.

Proof. Since $z$ satisfies the linear equation (3.5), we have, explicitly,

$$
D^{j} z(x, T)=\int \partial_{x}^{j} K(x, y, T) z(y, 0) d y .
$$

Since $|z(x, 0)| \leqslant M$ for all $x$, and $T>0$ is fixed, we simply estimate

$$
\left|D^{j} z(x, T)\right| \leqslant \delta_{1} \int_{|y|<R_{0}}\left|\partial_{x}^{j} K\right| d y+M \int_{|y|>R_{0}}\left|\partial_{x}^{j} K\right| d y .
$$

The lemma then follows if we can show that $\int_{|y|<R_{0}}\left|\partial_{x}^{j} K\right| d y \leqslant C$, independent of $R_{0}$ for $|x|<R_{1}$, and $\int_{|y|>R_{0}}\left|\partial_{x}^{j} K\right| d y \rightarrow 0$ as $R_{0} \rightarrow \infty$, uniformly for $|x|<R_{1}$. But both these estimates follow from the estimate

$$
\left|\partial_{x}^{j} K(x, y, T)\right| \leqslant \begin{cases}C(T) \exp \frac{-\left(|y|-R_{1}\right)^{2}}{4 T} \frac{\cosh y / 2}{1} & \text { if }|y|>R_{1}, \\ C\left(T, R_{1}\right) & \text { if }|y|<R_{1},\end{cases}
$$

valid for $|x| \leqslant R_{1}$. The function on the right side of (3.7) is clearly in $L^{1}$. Lemma 3.5 follows, finishing the proof of Proposition 3.3. 


\section{REFERENCES}

1. J. Bona and M. Schonbek, Traveling-wave solutions of the Korteweg-deVries-Burgers' equation, Proc. Roy. Soc. Edinburgh A (to appear).

2. J. L. Bona and M. Schonbek, Model equations for long waves with initial data corresponding to bore propagation (in preparation).

3. J. L. Bona and R. Smith, The initial value problem for the KdV equation, Philos. Trans. Roy. Soc. London Ser. A 278 (1975), 555-604.

4. A. Friedman, Partial differential equations of parabolic type, Prentice-Hall, Englewood Cliffs, N.J., 1964.

5. J. Goodman, Nonlinear asymptotic stability of viscous shock profiles for conservation laws, Courant Institute, preprint.

6. E. Hopf, The partial differential equation $u_{t}+u u_{x}=\mu u_{x x}$, Comm. Pure Appl. Math. 3 (1950), 201-230.

7. A. M. II'in and O. A. Oleinik, Asymptotic behavior of solutions of the Cauchy problem for some quasilinear equations for large time, Mat. Sb. 56 (93) (1960), 191-216. (Russian)

8. S. Klainerman and G. Ponce, Global small amplitude solutions to nonlinear evolution equations, Comm. Pure Appl. Math. 36 (1983), 133-141.

9. A. Majda and R. Pego, Stable viscosity matrices for systems of conservation laws, J. Differential Equations (to appear).

10. A. Matsumura and T. Nishida, The initial value problem for the equations of motion of compressible viscous and heat-conductive fluids, Proc. Japan Acad. Ser. A. Math. Sci. 55 (1979), 337-342.

11. L. A. Peletier, Asymptotic stability of traveling waves, Instability of Continuous Systems $(\mathrm{H}$. Leipholz, ed.), Springer, New York, 1971, pp. 418-422.

12. D. H. Sattinger, On the stability of waves of nonlinear parabolic systems, Adv. in Math. 22 (1976), $312-355$.

13. R. L. Pego, Stability in systems of conservation laws with dissipation (Seminar on Nonlinear PDE, Santa Fe, 1984), Lectures in Appl. Math. (to appear).

Department of Mathematics, University of Michigan, Ann Arbor, Michigan 48109 